\title{
Mangiferin inhibits hypoxia/reoxygenation-induced alveolar epithelial cell injury via the SIRT1/AMPK signaling pathway
}

\author{
XIANFENG CHEN and JUANJUAN HUANG \\ Department of Traditional Chinese Medicine, The Affiliated People's Hospital of \\ Ningbo University, Ningbo, Zhejiang 315040, P.R. China
}

Received June 17, 2021; Accepted July 26, 2021

DOI: $10.3892 /$ etm.2021.10654

\begin{abstract}
Lung ischemia-reperfusion injury (LIRI) is one of the complications that can occur after lung transplantation and may lead to morbidity and mortality. Mangiferin (MAF) is a naturally occurring glucosyl xanthone that has been documented to possess anti-inflammatory, immunomodulatory and potent antioxidant effects. The purpose of the present study was to investigate the effect of MAF on LIRI using a hypoxia-reoxygenation $(\mathrm{H} / \mathrm{R})$ cell model. In the present study, the viability of lung alveolar epithelial cells (A549) and H/R-A549 were detected by MTT assay. ELISA was used to evaluate the expression levels of IL- 6 and IL-1 $\beta$. TUNEL assay and western blotting were used to evaluate the apoptosis. In addition, H/R-A549 cells were treated with sirtinol, which is known inhibitor of sirtuin 1 (SIRT1) activity, to determine the effects of MAF on proteins associated with the SIRT1/5'AMP-activate protein kinase (AMPK) signaling pathway using western blotting. The results showed that $20 \mu \mathrm{M}$ MAF exerted a protective effect on A549 cells against $\mathrm{H} / \mathrm{R}$ mediating no clear cytotoxic effects. In terms of inflammation, MAF reduced IL-6, IL-1 $\beta$, cyclooxygenase-2 and inducible nitric oxide synthase expression, which was accompanied by activation of the SIRT1/AMPK signaling pathway. In addition, compared with those in the group treated with sirtinol, expression of SIRT1, Bcl-2 and AMPK activity were elevated in MAF-treated H/R-A549 cells, whereas the expression of Bax, cleaved caspase- 3 and cleaved caspase- 9 was suppressed. TUNEL analysis of H/R-A549 cells treated with MAF in combination with sirtinol revealed that treatment with sirtinol blocked the SIRT1/AMPK signaling pathway and increased the apoptosis rate compared with the MAF group. Taken together, results of the present study revealed that MAF
\end{abstract}

Correspondence to: Dr Xianfeng Chen, Department of Traditional Chinese Medicine, The Affiliated People's Hospital of Ningbo University, 251 Baizhang East Road, Ningbo, Zhejiang 315040, P.R. China

E-mail: chenxianfeng011@126.com

Key words: mangiferin, hypoxia/reoxygenation, lung injury, sirtuin 1,5'AMP-activated protein kinase could inhibit lung H/R cell injury through the SIRT1/AMPK signaling pathway.

\section{Introduction}

Lung ischemia-reperfusion injury (LIRI) is one of the complications that can potentially arise after lung transplantation (1). Following lung transplantation, organ ischemia and subsequent reperfusion is unavoidable and frequently leads to acute sterile inflammation in a process known as ischemia reperfusion injury $(1,2)$. LIRI may also develop into pulmonary infection, acute lung injury and bronchiolitis obliterans syndrome, thereby reducing the post-transplant survival rate and increasing risk of mortality (3).

Sirtuins (SIRT) belong to a protein family that is responsible for regulating various intracellular events, including cell proliferation, apoptosis and cell migration $(4,5)$. In particular, sirtuin 1 (SIRT1) is one of the most studied isoform of the sirtuin family $(6,7)$. SIRT1 is also known as a NAD ${ }^{+}$-dependent histone deacetylase and has been revealed to be an important modulator of energy metabolism $(6,7)$. Notably, it has been previously shown that LIRI can be alleviated by activating SIRT (8-10).

5'AMP-activated protein kinase (AMPK) is an ubiquitous energy sensor enzyme and is considered to be a downstream effector of SIRT1, which serves a key role in the regulation of energy homeostasis and cell survival $(4,11,12)$. AMPK has been reported to exert SIRT1-dependent anti-inflammatory activities in sepsis-induced acute lung injury (13).

Over the past decade, there has been growing interest in the potential application of natural bioactive components isolated from plants for therapeutic uses. Mangiferin (MAF) is a C-glucosyl xanthone that is present at high levels in higher plants such as mango (Mangifera indica L.) $(14,15)$. It has been demonstrated to possess numerous pharmacological activities, including antiviral, anticancer, antioxidative, antiaging, immunomodulatory and analgesic effects (15-18). Furthermore, it has been reported that MAF can prevent liver lipid metabolism disorders by regulating the SIRT1/AMPK pathway (19) whilst also inhibiting pulmonary fibrosis (20). However, the effects of MAF on LIRI remains poorly understood. Therefore, the aim of the present study was to evaluate the effects of MAF on LIRI using an in vitro hypoxia/reoxygenation $(\mathrm{H} / \mathrm{R})$ cell model and explore its possible mechanism. 


\section{Materials and methods}

Cell culture and modeling. The human alveolar epithelial cells (A549) were obtained from American Type Culture Collection (ATCC). They were routinely cultured in RPMI-1640 medium supplemented with $10 \%$ fetal bovine serum (FBS) (both from Beijing Solarbio Science \& Technology Co., Ltd.), 100 U/ml penicillin and $100 \mathrm{pg} / \mathrm{ml}$ streptomycin in a humidified incubator with $5 \% \mathrm{CO}_{2}$ at $37^{\circ} \mathrm{C}$.

A H/R-A549 cell model was established. Briefly, A549 cells were exposed to anoxia for $24 \mathrm{~h}\left(1 \% \mathrm{O}_{2}, 5 \% \mathrm{CO}_{2}\right.$ and $\left.94 \% \mathrm{~N}_{2}\right)$ at $37^{\circ} \mathrm{C}$ followed by $4 \mathrm{~h}$ of reoxygenation $\left(5 \% \mathrm{CO}_{2}\right.$ and $95 \%$ air) at $37^{\circ} \mathrm{C}(\mathrm{H} / \mathrm{R}$ group) $(21,22)$.

The cell model with the SIRT1/AMPK signaling pathway blocked (H/R-A549 + sirtinol) was established. Sirtinol stock solution (10 mM) (Sigma-Aldrich; Merck KGaA) and MAF (Sigma-Aldrich; Merck KGaA; Fig. 1A) stock solution $(50 \mathrm{mM})$ were prepared in dimethyl sulfoxide and stored at $-80^{\circ} \mathrm{C}$ until further use. H/R-A549 cells were treated with $15 \mu \mathrm{M}$ of sirtinol for $72 \mathrm{~h}$ at $37^{\circ} \mathrm{C}(23)$. Subsequently, the cells were cultured for another $24 \mathrm{~h}$ with $20 \mu \mathrm{M} \mathrm{MAF}$ at $37^{\circ} \mathrm{C}(\mathrm{H} / \mathrm{R}$ + MAF + sirtinol group). Additionally, H/R-A549 cells were incubated with $20 \mu \mathrm{M}$ MAF at $37^{\circ} \mathrm{C}$ for $24 \mathrm{~h}(\mathrm{H} / \mathrm{R}+\mathrm{MAF}$ group).

Cell viability test. Cell viability was measured using MTT assay. A549 and H/R-A549 cells (5x103/well) were each incubated in 96-well plates with various concentrations of MAF $(5,10,20$ and $50 \mu \mathrm{M})$. Using the RPMI-1640 medium to dilute the drug stock solution, the cell toxicity of DMSO was negligible due to the use of $\geq 1,000$-fold dilution. After incubation for $24 \mathrm{~h}, 20 \mu 1 \mathrm{MTT}(5 \mathrm{mg} / \mathrm{ml})$ was added to each well and cells were incubated for a further $4 \mathrm{~h}$ at $37^{\circ} \mathrm{C}$. A buffer solution (10\% SDS, $5 \%$ isopropanol and $0.1 \% \mathrm{HCl}$ ) was then added to solubilize the MTT formazan crystals overnight. The absorbance in each well was measured at $570 \mathrm{~nm}$ using a microplate reader (Thermo Fisher Scientific, Inc.). Untreated cells were used as a control.

ELISA cytokine assay. A549 and H/R-A549 cells (5x10/well) were inoculated into 96-well plates and treated with different MAF concentrations $(5,10$ and $20 \mu \mathrm{M})$ for $24 \mathrm{~h}$ at $37^{\circ} \mathrm{C}$. Subsequently, the cell culture medium was centrifuged at $2,000 \mathrm{x} \mathrm{g}$ for $4 \mathrm{~min}$ at $4^{\circ} \mathrm{C}$ to remove debris and the supernatant was collected for assay. The levels of IL-6, IL-1 $\beta$ and IL-10 in cells were quantified using their corresponding ELISA kits (cat. no. E-EL-H0102c, E-EL-H0149c and E-EL-H0103c; Elabscience Biotechnology Co., Ltd.) according to the manufacturer's protocols. The absorbance at $450 \mathrm{~nm}$ was measured using a microplate reader (Thermo Fisher Scientific, Inc.).

TUNEL assay. Apoptotic A549 and H/R-A549 cells were visualized using one-step TUNEL apoptosis detection kit according to the manufacturer's protocol (cat. no. KGA7071; Nanjing KeyGen Biotech Co., Ltd.). Briefly, cells $\left(2 \times 10^{6} / \mathrm{ml}\right)$ that were cultured on cover slips were fixed using $4 \%$ neutral-buffered formalin solution at room temperature for $30 \mathrm{~min}$ and incubated with $0.3 \%$ Triton X-100 at room temperature for $5 \mathrm{~min}$. Subsequently, each sample was supplemented with $50 \mu$ l TUNEL detection reagent for $60 \mathrm{~min}$ at $37^{\circ} \mathrm{C}$ in the dark.
The cell nuclei were stained with $5 \mu \mathrm{g} / \mathrm{ml}$ DAPI at $37^{\circ} \mathrm{C}$ in the dark for $5 \mathrm{~min}$. An anti-fade solution (Beijing Solarbio Science \& Technology Co., Ltd.) was dropped onto the area containing the treated cells and the sections were mounted onto the slides. Finally, all samples were imaged in three random fields per coverslip using a fluorescence microscope (magnification, $\mathrm{x} 100$ ) to view the green fluorescence at $520 \pm 20 \mathrm{~nm}$ and blue DAPI at $460 \mathrm{~nm}$.

Western blotting assay. The protein samples from the cells were extracted using the cell lysis buffer (cat. no. P0013; Beyotime Biotechnology Inc.) and the protein content was measured using a BCA Protein Assay Kit (cat. no. P0012S; Beyotime Biotechnology Inc.). The protein samples ( $40 \mu \mathrm{g})$ were subjected to $15 \%$ SDS-PAGE for separation and transferred onto nitrocellulose membranes. After blocking in fresh $5 \%$ non-fat milk at room temperature for $2 \mathrm{~h}$, the membranes were incubated overnight at $4^{\circ} \mathrm{C}$ with primary antibodies, followed by incubation with a HRP-conjugated secondary antibody at room temperature for $2 \mathrm{~h}$. An enhanced chemiluminescence (ECL) kit (Thermo Fisher Scientific, Inc.) was used to visualize the signals. The antibodies (Affinity Biosciences) used included: Anti-SIRT1 (cat. no. DF6033; 1:1,000), anti-phosphorylated (p-)-AMPK (cat. no. AF3423; 1:1,000), anti-AMPK (cat. no. AF6423; 1:1,000), anti-Cox-2 (cat. no. AF7003; 1:1,000), anti-iNOS (cat. no. AF0199; 1:1,000), anti-Bcl-2 (cat. no. AF6139; 1:1,000), anti-Bax (cat. no. AF0120; 1:1,000), anti-cleaved-caspase-3 (cat. no. AF7022; 1:1,000), anti-cleaved-caspase-9 (cat. no. AF5240; 1:1,000), anti-GAPDH (cat. no. AF7021; $1: 5,000)$ and the goat anti-rabbit IgG secondary antibody (cat. no. S0001; 1:5,000). Protein expression levels were semi-quantified using Image-Pro Plus software version 6.0 (Media Cybernetics, Inc.).

Statistical analyses. All data were expressed as the mean \pm standard deviation. statistical analysis was performed with GraphPad Prism 8.0 software (GraphPad Software, Inc.). Differences between the means of the groups were compared using a one-way ANOVA followed by Tukey's test. Each experiment was repeated $\geq$ three times. $\mathrm{P}<0.05$ was considered to indicate a statistically significant difference.

\section{Results}

MAF promotes the viability of $H / R-A 549$ cells. As shown in Fig. 1B, MAF at concentrations of $<50 \mu \mathrm{M}$, could not exert toxicity on A549 cells. However. $50 \mu \mathrm{M}$ MAF was found to be significantly toxic to cells. According to Fig. 1C, cell viability after H/R induction was significantly decreased compared with that in the control group. By contrast, the viability of H/R-A549 cells was increased significantly after treatment with $20 \mu \mathrm{M}$ MAF. However, after treatment with $50 \mu \mathrm{M}$ MAF, cell viability was markedly decreased compared with that in the $20 \mu \mathrm{M}$ MAF treatment group. Therefore, in the subsequent experiments, the maximum concentration of MAF used was $20 \mu \mathrm{M}$.

MAF inhibits inflammation in H/R-A549 cells. The levels of the inflammatory markers in H/R-A549 cells treated with 
A<smiles>O=c1c2cc(O)c(O)cc2oc2cc(O)c(O)c(O)c12</smiles>

B

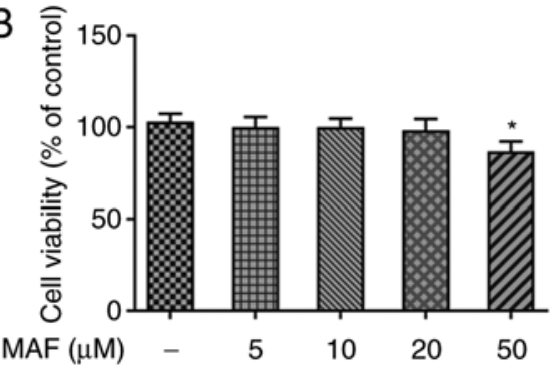

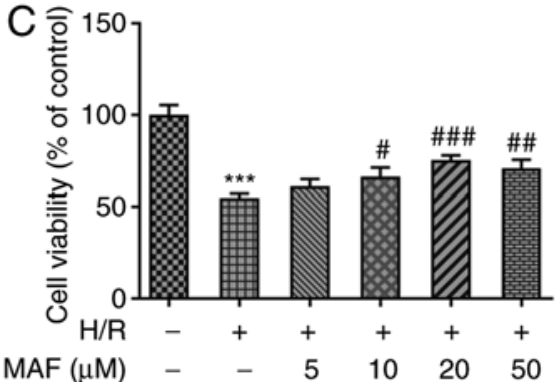

Figure 1. Effect of MAF on A549 cell viability. (A) Chemical structure of MAF. Cell viability of (B) A549 and (C) H/R-A549 after MAF treatment as detected using the MTT method. ${ }^{\mathrm{P}}<0.05$ and ${ }^{* * *} \mathrm{P}<0.001$ vs. Control; ${ }^{\#} \mathrm{P}<0.05,{ }^{\# \#} \mathrm{P}<0.01$ and ${ }^{\# \# \#} \mathrm{P}<0.001$ vs. H/R. MAF, mangiferin; H/R, hypoxia-reoxygenation.
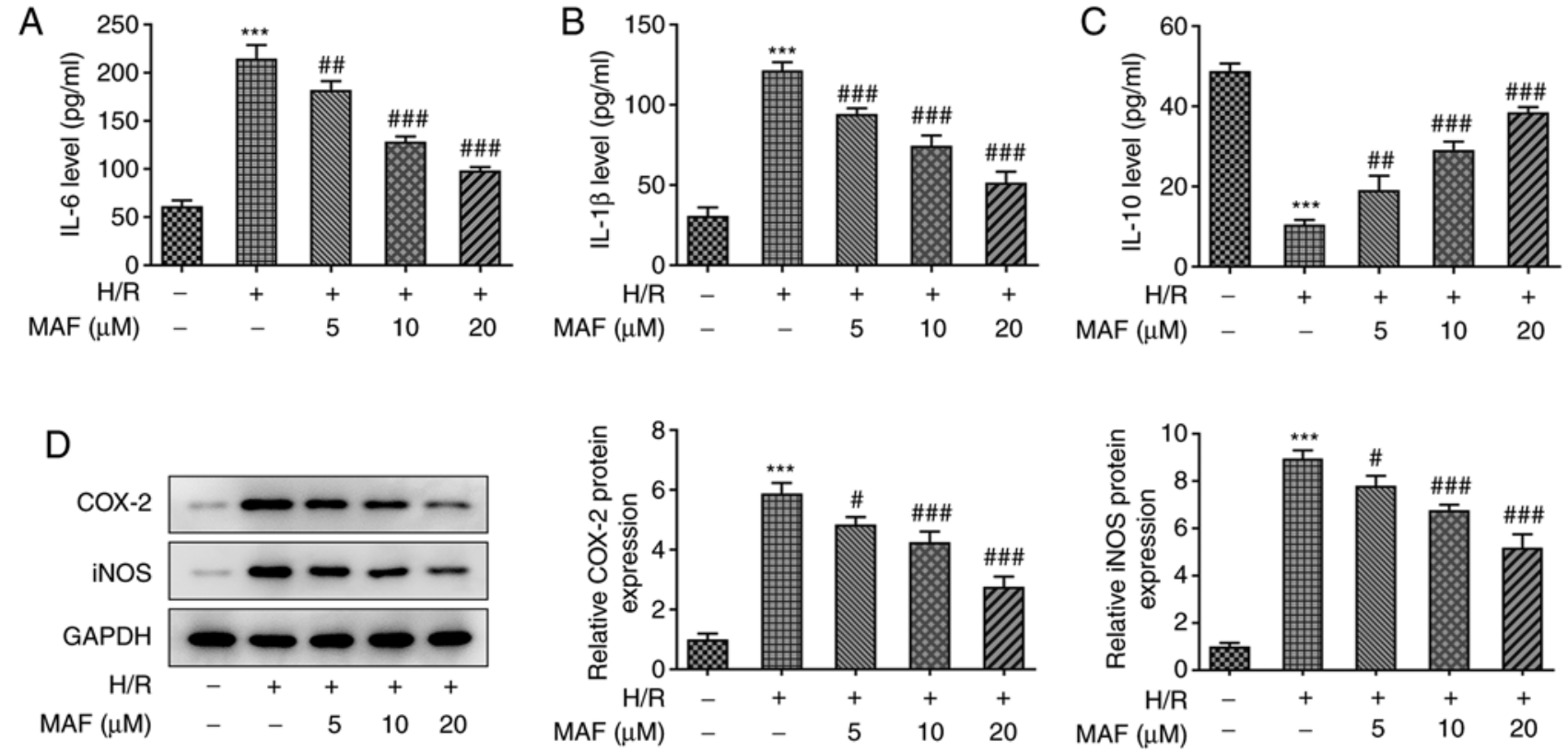

Figure 2. Effect of MAF on inflammation induced by H/R. The levels of (A) IL-6, (B) IL-1 $\beta$ and (C) IL-10 in H/R-A549 cells treated with different concentrations of MAF were detected by ELISA. (D) Western blot analysis of Cox-2 and iNOS protein expression in H/R-A549 cells treated with different concentrations of MAF. ${ }^{* * *} \mathrm{P}<0.001$ vs. Control; ${ }^{\#} \mathrm{P}<0.05,{ }^{\# \#} \mathrm{P}<0.01$ and ${ }^{\# \# \#} \mathrm{P}<0.001$ vs. H/R. MAF, mangiferin; H/R, hypoxia-reoxygenation; Cox-2, cyclooxygenase-2; iNOS, inducible nitric oxide synthase.

different concentrations of MAF were measured. As shown in Fig. 2A-C, ELISA analysis showed that compared with those in the control group, H/R significantly upregulated the expression levels of IL- 6 and IL- $1 \beta$, whilst significantly downregulating the expression level of IL-10. By contrast, the H/R-induced upregulation of IL-6 and IL-1 $\beta$ and downregulation of IL-10 were significantly reversed in the MAF-treated groups in a dose-dependent manner. In addition, the protein expression levels of Cox- 2 and iNOS were also significantly increased in the $\mathrm{H} / \mathrm{R}$ induced group compared with those in the control group, whilst those in the MAF-treated groups were significantly reversed compared with those in the H/R group. Taken together, these data suggest that MAF treatment can inhibit the-H/R induced inflammatory response.

$M A F$ inhibits apoptosis in H/R-A549 cells. Nuclear DNA fragmentation is an important biochemical event during apoptosis in many cell types and was therefore measured by TUNEL assay in the present study. The TUNEL results showed that A549 cell apoptosis was markedly increased after H/R induction (Fig. 3A). By contrast, after the addition of MAF, the H/R-induced cell apoptosis was reduced (Fig. 3A). In addition, compared with those in the control group, the expression levels of Bax, cleaved-caspase- 3 and cleaved-caspase 9 protein were significantly increased in the $\mathrm{H} / \mathrm{R}$ group, whilst those of Bcl-2 were reduced (Fig. 3B). Notably, the expression level of Bcl-2 was significantly increased by 10 and $20 \mu \mathrm{M}$ MAF treatment compared with that in the H/R group (Fig. 3B). In addition, the expression levels of Bax, cleaved-caspase-3 and cleaved-caspase-9 were significantly decreased in $20 \mu \mathrm{M}$ MAF treatment group compared with those in the $H / R$ group (Fig. 3B). These results suggest that MAF can inhibit $\mathrm{H} / \mathrm{R}$-induced apoptosis in A549 cells.

MAF activates the SIRTI/AMPK signaling pathway. To verify the hypothesis that MAF may serve a role in the SIRT1/AMPK signaling pathway, the protein levels of SIRT1 and ratio of $\mathrm{p}$-AMPK/AMPK were evaluated in H/R cells by western blotting assay. As shown in Fig. 4, compared with those in the control group, the protein expression of 
A
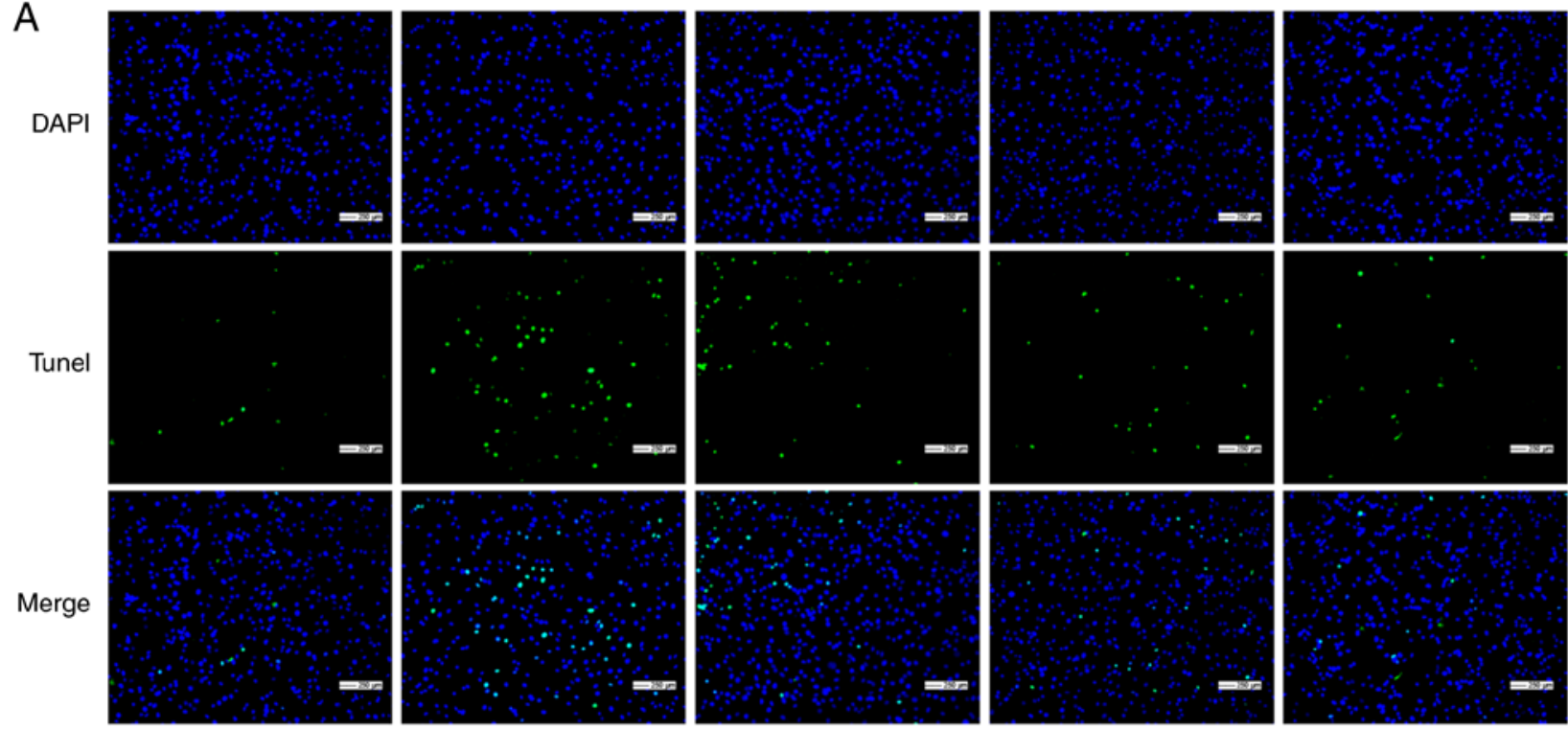

$\operatorname{MAF}(\mu \mathrm{M})$

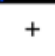

5

10

20

B
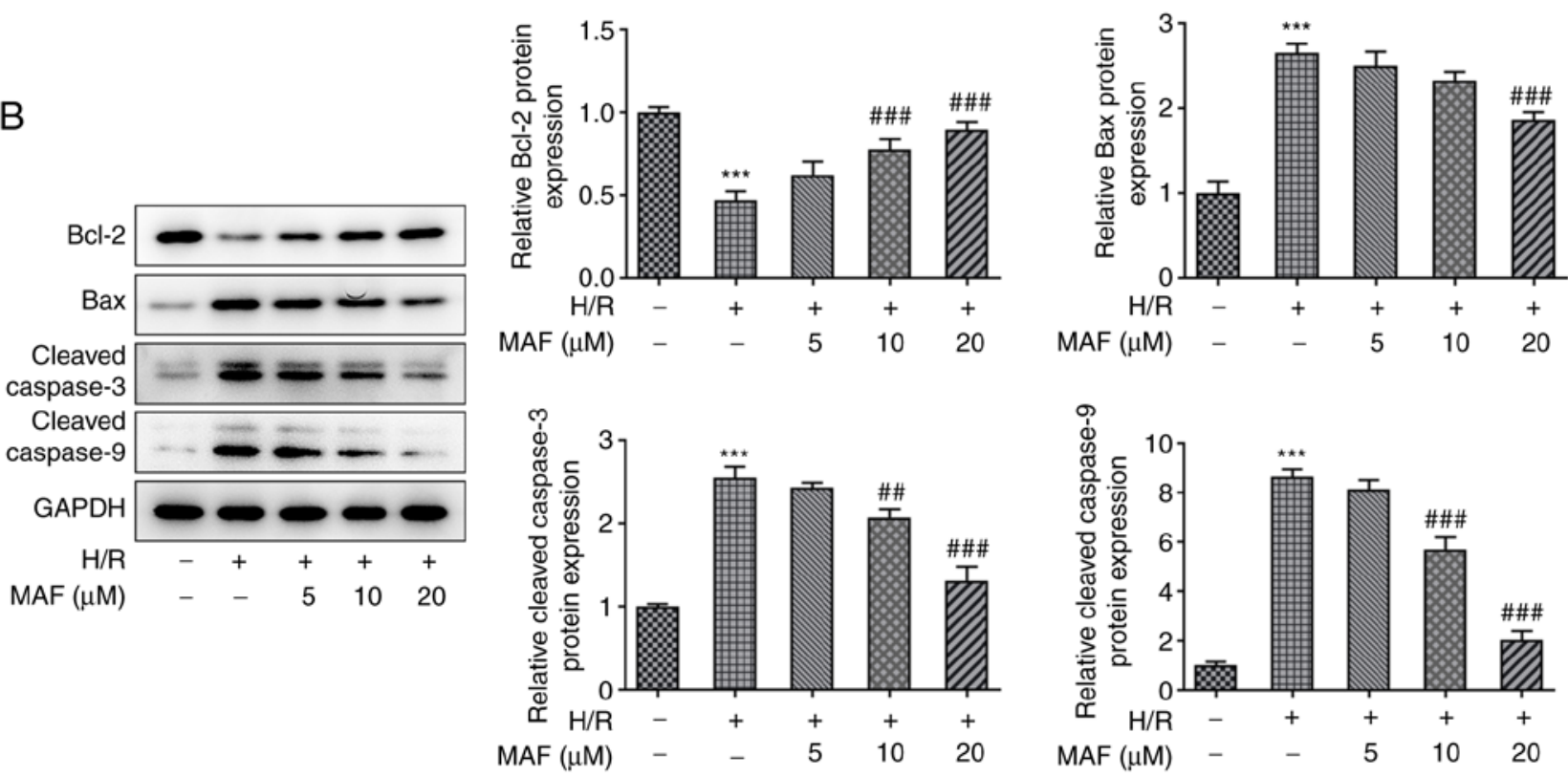

Figure 3. Effect of MAF on apoptosis induced by H/R. (A) Representative TUNEL images in each group after treatment with various concentrations of MAF. Scale bars, $250 \mu \mathrm{m}$. (B) Western blot analysis and quantification of Bcl-2, Bax, cleaved-caspase-3 and cleaved-caspase- 9 in MAF treatment.

${ }^{* * * *} \mathrm{P}<0.001$ vs. Control; ${ }^{\# \prime} \mathrm{P}<0.01$ and ${ }^{\# \# \#} \mathrm{P}<0.001$ vs. H/R. MAF, mangiferin; H/R, hypoxia-reoxygenation.

SIRT1 and ratio of p-AMPK/AMPK were significantly deceased in the H/R-induced group. By contrast, compared with the H/R-induced group, the protein levels of SIRT1 and ratio p-AMPK/AMPK were significantly increased in the 10 and $20 \mu \mathrm{M}$ MAF treatment groups (Fig. 4). Since a concentration of $20 \mu \mathrm{M}$ MAF resulted in a highly significant increase in SIRT1 and p-AMPK/AMPK levels, $20 \mu \mathrm{M}$ MAF was used subsequently to explore the effects of MAF on the SIRT1/AMPK signaling pathway.

MAF inhibits H/R injury in cells through the SIRTI/AMPK signaling pathway. To understand whether the SIRT1/AMPK signaling pathway affected $\mathrm{H} / \mathrm{R}$-induced inflammation and apoptosis, the SIRT1 inhibitor sirtinol was used to intercept the SIRT1/AMPK signaling pathway following MAF treatment. As shown in Fig. 5A-D, after blocking the SIRT1/AMPK signaling pathway, the protective effects of $20 \mu \mathrm{M}$ MAF against inflammation were reversed. Compared with those in the MAF treatment group (H/R + MAF), the pathway inhibition group $(H / R+M A F+$ sirtinol) significantly upregulated the expression levels of IL- 6 and IL-1 $\beta$, whilst significantly downregulating the expression level of IL-10 (Fig. 5A-C). In addition, the protein expression levels of Cox- 2 and iNOS were also significantly increased in the $\mathrm{H} / \mathrm{R}+\mathrm{MAF}+$ sirtinol group compared with those in the H/R + MAF group (Fig. 5D). After inhibiting the SIRT1/AMPK signaling pathway, the rate of apoptosis in MAF treatment was markedly increased compared with that in the H/R + MAF group (Fig. 5E). Compared with 

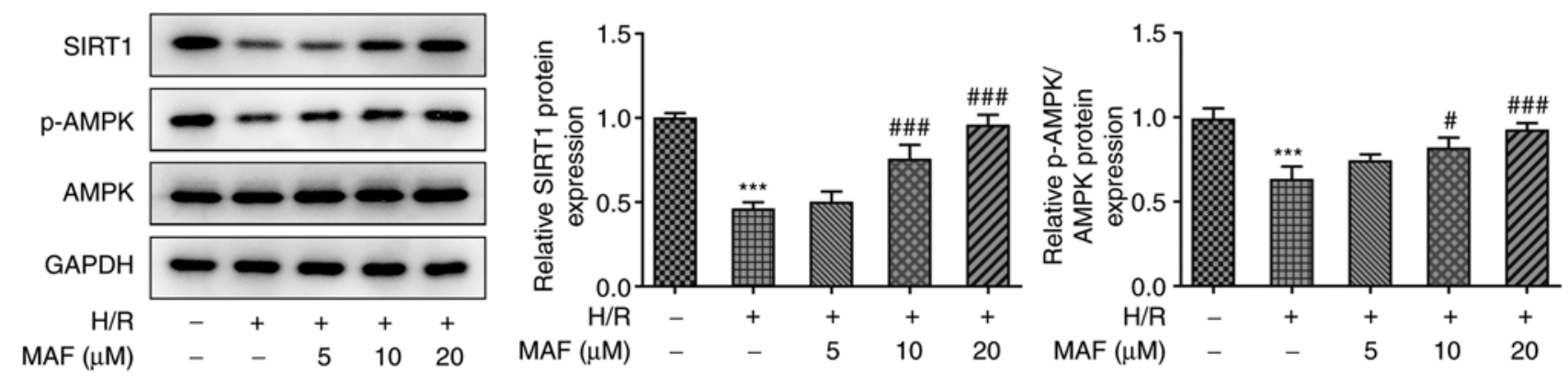

Figure 4. Western blot analysis and quantification of SIRT1 expression and AMPK activation in alveolar epithelial cells and H/R cells after treatment with different concentrations of MAF. ${ }^{* * *} \mathrm{P}<0.001$ vs. control; $\mathrm{P}<0.05$ and ${ }^{\# \# \#} \mathrm{P}<0.001$ vs. H/R. MAF, mangiferin; H/R, hypoxia-reoxygenation; SIRT1, sirtuin 1; AMPK, 5'AMP-activated protein kinase.
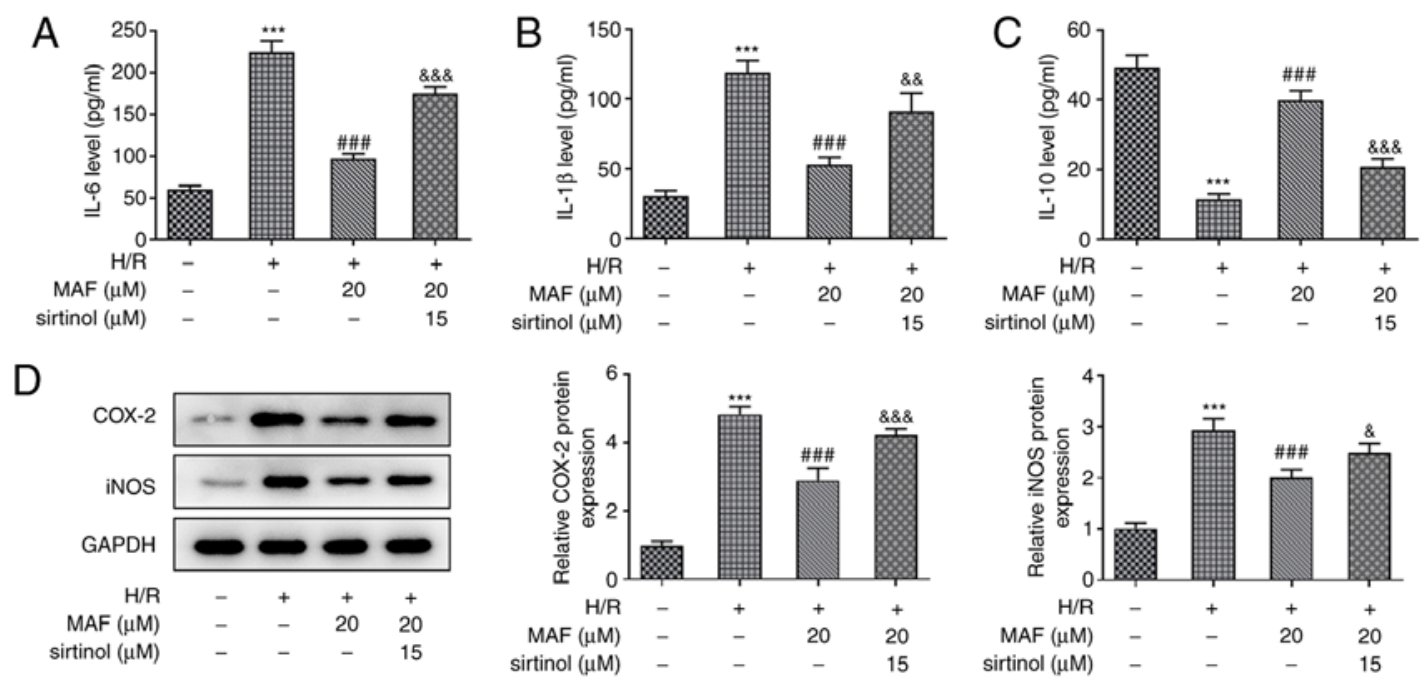

E
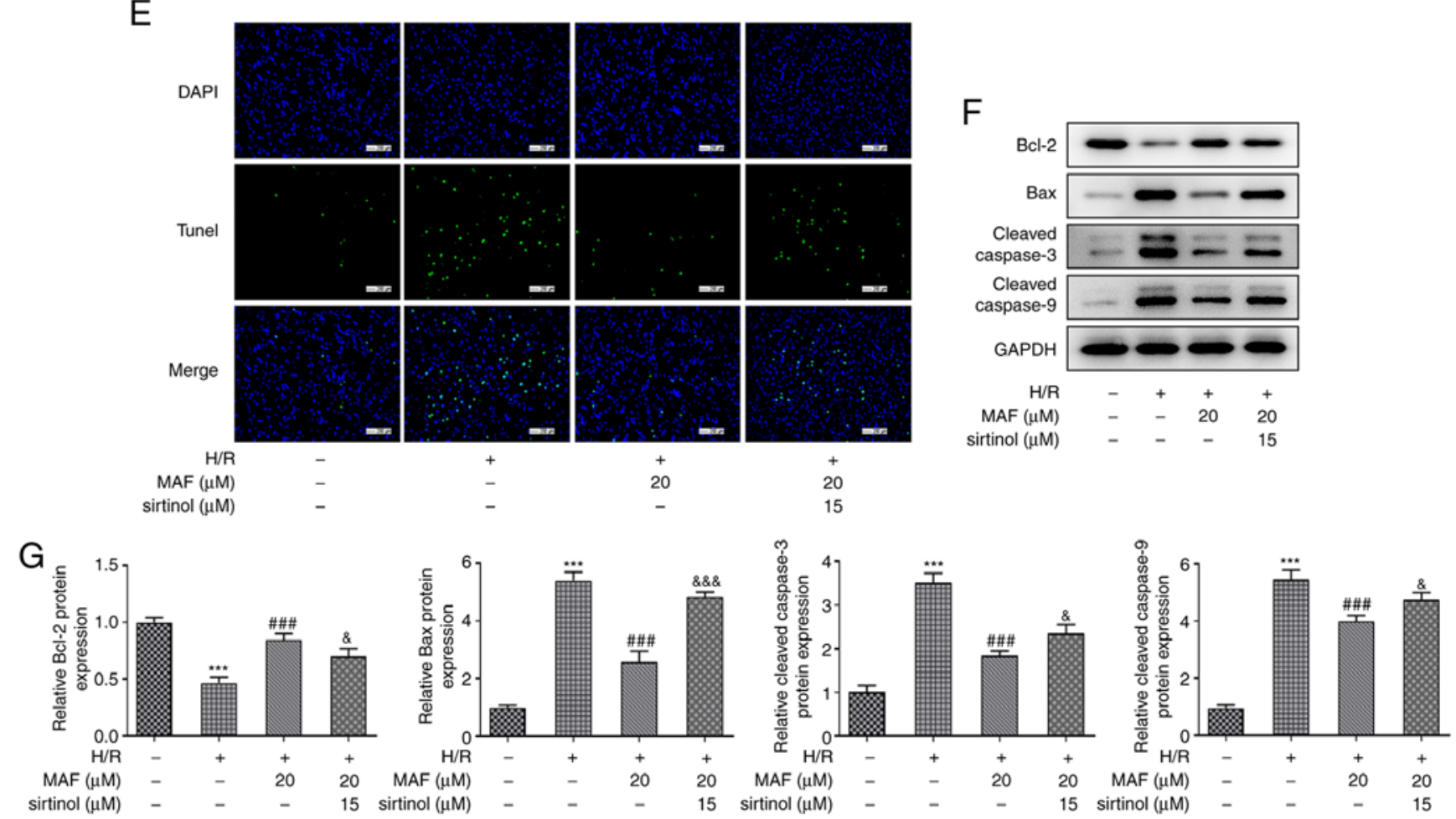

Figure 5. Effect of MAF and sirtinol on H/R-induced cell injury and the status of the sirtuin 1/5'AMP-activated protein kinase signaling pathway. The levels of (A) IL-6, (B) IL-1 $\beta$ and (C) IL-10 in H/R-A549 cells were detected by ELISA. (D) Western blot analysis of Cox-2 and iNOS protein expression in H/R-A549 cells. (E) Representative TUNEL images of alveolar epithelial cells. Scale bars, $250 \mu \mathrm{m}$. (F) Western blot analysis and (G) statistical quantification of Bcl-2, Bax, cleaved-caspase-3 and cleaved-caspase-9. ${ }^{* * *} \mathrm{P}<0.001$ vs. Control. ${ }^{\# \# "} \mathrm{P}<0.001$ vs. H/R. ${ }^{\&} \mathrm{P}<0.05$, ${ }^{\text {\& }} \mathrm{P}<0.01$ and ${ }^{\text {\&\&\& }} \mathrm{P}<0.001$ vs. H/R + MAF. MAF, mangiferin; H/R, hypoxia-reoxygenation; Cox-2, cyclooxygenase-2; iNOS, inducible nitric oxide synthase. 
those in the MAF treatment group, the protein expression levels of Bax, cleaved-caspase-3 and cleaved-caspase-9 were significantly increased after the addition of sirtinol, whereas Bcl-2 expression was significantly reduced (Fig. 5F). Altogether, these results suggest that MAF can activate the SIRT1/AMPK signaling pathway to inhibit H/R-induced A549 cell injury.

\section{Discussion}

The success of lung transplantation was limited by the high rates of primary graft dysfunction due to ischemia-reperfusion injury, which is characterized by potent inflammation, alveolar damage and vascular permeability $(1,24)$. Ischemia-reperfusion injury that occurs following lung transplantation typically activates the innate immune system to induce inflammation $(1,9)$. In turn, this inflammation can enhance acute allograft rejection, impair transplant tolerance and accelerate the progression of chronic rejection (25). A number of reports in cellular and animal models have shown that the levels of inflammatory factors, including IL- 6 and IL-1 $\beta$, were significantly increased after lung injury, whereas those of immunosuppressive factors, including IL-10, were decreased $(26,27)$. Therefore, it is necessary to reduce the occurrence of inflammation to mitigate the damage induced by LIRI.

In recent years, the potential application of naturally occurring components of plants for clinical uses has attracted much attention (28-30). MAF is a natural constituent of foods and traditional herbal medicines, such as Mangifera indica, Anemarrhena asphodeloides and Coffea pseudozanguebariae, that exhibits almost no adverse effects or toxicity (31). In addition, it possesses numerous pharmacological activities, such as anti-inflammatory, immunomodulatory and antioxidative effects (15). In the present study, MAF exerted little to no toxicity on A549 cells but mediated protective effects on cells after H/R injury, which was similar to previous reports that MAF attenuated myocardial ischemia-reperfusion injury and lung injury $(32,33)$. When the concentration of MAF used was $<20 \mu \mathrm{M}, \mathrm{H} / \mathrm{R}$ injury was protected by MAF in a dose-dependent manner.

A previous study showed that MAF could improve cell viability after $H / R$ injury, which clarified the protective effects of MAF against myocardial injury (32). In addition, on inflammation, Wang et al found that MAF pretreatment significantly inhibits ischemia-reperfusion-induced elevated expression levels of TNF- $\alpha$ and IL-1 $\beta$ (34). Similarly, in the present study, the increased levels of IL- 6 and IL-1 $\beta$ induced by H/R were markedly inhibited after MAF pretreatment. Previous studies have demonstrated that MAF can suppress apoptosis by reducing the protein expression levels of cleaved-caspase-3, caspase-9 and Bax whilst increasing those of Bcl-2 (35-37). Likewise, the apoptosis data found in the present study showed that MAF downregulated the expression levels of Bax, cleaved-caspase- 3 and cleaved-caspase- 9 protein and increased the expression of $\mathrm{Bcl}-2$ to reduce apoptosis, consistent with the previously reported data aforementioned. Therefore, based on these data, the present study suggests that MAF exerted anti-inflammatory and anti-apoptotic effects on A549 cells.

Mechanistically, MAF exerts anti-inflammatory effects by activating the SIRT1 signaling pathway $(38,39)$. Various studies have revealed that SIRT1 is a key component in a number of stress-related pathways, including cell apoptosis, cellular senescence and angiogenesis $(40,41)$. In addition, SIRT1 has been proposed to be an attractive therapeutic target for myocardial ischemia-reperfusion injury $(42,43)$. SIRT1 activation can inhibit the cardiac inflammatory response following ischemia-reperfusion (44). Once activated, SIRT1 can mediate a wide range of downstream signaling pathways, such as AMPK, peroxisome proliferator-activated receptor $\gamma$ coactivator $1 \alpha$ and endothelial nitric oxide synthase $(39,42,45)$. In the present study, the protein expression of SIRT1 and AMPK was increased in a dose-dependent manner after MAF treatment. After the addition of sirtinol, a SIRT1 inhibitor, the anti-inflammatory and anti-apoptotic effects of MAF were reversed. Therefore, MAF can activate the SIRT1/AMPK signaling pathway. Taken together, activation of the SIRT1/AMPK signaling pathway confer protective effects against ischemia-reperfusion injury.

In the present study, the effects of MAF were evaluated on an in vitro A549 cell model of LIRI using a cellular H/R model. The results showed that MAF could induce the activation of the SIRT1/AMPK signaling pathway, where it protected A549 cell injury induced by H/R by inhibiting inflammation and apoptosis. Application of MAF therefore provides a promising strategy for the treatment of LIRI. However, the fact that the present study only used in vitro and not in vivo models and examined the effects of MAF concentration on inflammation and apoptosis are limitations of the present study. In addition, further elucidation in clinical samples is required.

\section{Acknowledgements}

Not applicable.

\section{Funding}

No funding was received.

\section{Availability of data and materials}

The datasets used and/or analyzed during the current study are available from the corresponding author on reasonable request.

\section{Authors' contributions}

$\mathrm{XC}$ and $\mathrm{JH}$ conceived and designed the study, acquired and interpreted the data and revised it for critically important intellectual content. XC and JH confirm the authenticity of all raw data in the present study. All authors have read and approved the final manuscript.

\section{Ethics approval and consent to participate}

Not applicable.

\section{Patient consent for publication}

Not applicable.

\section{Competing interests}

The authors declare that they have no competing interests. 


\section{References}

1. Laubach VE and Sharma AK: Mechanisms of lung ischemia-reperfusion injury. Curr Opin Organ Transplant 21: 246-252, 2016

2. Saito M, Chen-Yoshikawa TF, Suetsugu K, Okabe R, Takahagi A, Masuda S and Date H: Pirfenidone alleviates lung ischemia-reperfusion injury in a rat model. J Thorac Cardiovasc Surg 158: 289-296, 2019.

3. Shah RJ and Diamond JM: Primary graft dysfunction (PGD) following lung transplantation. Semin Respir Crit Care Med 39: 148-154, 2018.

4. You J, Cheng J, Yu B, Duan C and Peng J: Baicalin, a Chinese herbal medicine, inhibits the proliferation and migration of human non-small cell lung carcinoma (NSCLC) Cells, A549 and H1299, by activating the SIRT1/AMPK signaling pathway. Med Sci Monit 24: 2126-2133, 2018.

5. Jin MS, Hyun CL, Park IA, Kim JY, Chung YR, Im SA, Lee KH, Moon HG and Ryu HS: SIRT1 induces tumor invasion by targeting epithelial mesenchymal transition-related pathway and is a prognostic marker in triple negative breast cancer. Tumour Biol 37: 4743-4753, 2016.

6. Suzuki K, Hayashi R, Ichikawa T, Imanishi S, Yamada T, Inomata M, Miwa T, Matsui S, Usui I, Urakaze M, et al: SRT1720, a SIRT1 activator, promotes tumor cell migration, and lung metastasis of breast cancer in mice. Oncol Rep 27: 1726-1732, 2012.

7. Lovaas JD, Zhu L, Chiao CY, Byles V, Faller DV and Dai Y: SIRT1 enhances matrix metalloproteinase-2 expression and tumor cell invasion in prostate cancer cells. Prostate 73: 522-530, 2013.

8. Liu Z, Meng Y, Miao Y, Yu L and Yu Q: Propofol reduces renal ischemia/reperfusion-induced acute lung injury by stimulating sirtuin 1 and inhibiting pyroptosis. Aging (Albany NY) 13: $865-876,2020$

9. Yang C, Yang W, He Z, He H, Yang X, Lu Y and Li H: Kaempferol improves lung ischemia-reperfusion injury via antiinflammation and antioxidative stress regulated by SIRT1/HMGB1/NF- $\mathrm{B}$ axis. Front Pharmacol 10: 1635, 2019.

10. Fu C, Hao S, Xu X, Zhou J, Liu Z, Lu H, Wang L, Jin W and Li S: Activation of SIRT1 ameliorates LPS-induced lung injury in mice via decreasing endothelial tight junction permeability Acta Pharmacol Sin 40: 630-641, 2019.

11. Salomone F, Barbagallo I, Godos J, Lembo V, Currenti W, Cinà D, Avola R, D'Orazio N, Morisco F, Galvano F and Li Volti G: Silibinin restores NAD ${ }^{+}$levels and induces the SIRT1/AMPK pathway in non-alcoholic fatty liver. Nutrients 9: $1086,2017$.

12. Mulchandani N, Yang WL, Khan MM, Zhang F, Marambaud P, Nicastro J, Coppa GF and Wang P: Stimulation of brain AMP-activated protein kinase attenuates inflammation and acute lung injury in sepsis. Mol Med 21: 637-644, 2015.

13. Li X, Jamal M, Guo P, Jin Z, Zheng F, Song X, Zhan J and Wu H: Irisin alleviates pulmonary epithelial barrier dysfunction in sepsis-induced acute lung injury via activation of AMPK/SIRT1 pathways. Biomed Pharmacother 118: 109363, 2019.

14. Ajila CM, Rao LJ and Rao UJ: Characterization of bioactive compounds from raw and ripe Mangifera indica L. peel extracts. Food Chem Toxicol 48: 3406-3411, 2010.

15. Du S, Liu H, Lei T, Xie X, Wang H, He X, Tong R and Wang Y Mangiferin: An effective therapeutic agent against several disorders (Review). Mol Med Rep 18: 4775-4786, 2018.

16. Dar A, Faizi S, Naqvi S, Roome T, Zikr-ur-Rehman S, Ali M, Firdous S and Moin ST: Analgesic and antioxidant activity of mangiferin and its derivatives: The structure activity relationship. Biol Pharm Bull 28: 596-600, 2005.

17. Duang XY, Wang Q, Zhou XD and Huang DM: Mangiferin: A possible strategy for periodontal disease to therapy. Med Hypotheses 76: 486-488, 2011.

18. Imran M, Arshad MS, Butt MS, Kwon JH, Arshad MU and Sultan MT: Mangiferin: A natural miracle bioactive compound against lifestyle related disorders. Lipids Health Dis 16: 84, 2017.

19. Li J, Liu M, Yu H, Wang W, Han L, Chen Q, Ruan J, Wen S, Zhang Y and Wang T: Mangiferin improves hepatic lipid metabolism mainly through its metabolite-norathyriol by modulating SIRT-1/AMPK/SREBP-1c signaling. Front Pharmacol 9: 201, 2018.
20. Jia L, Sun P, Gao H, Shen J, Gao Y, Meng C, Fu S, Yao H and Zhang G: Mangiferin attenuates Bleomycin-induced pulmonary fibrosis in mice through inhibiting TLR4/p65 and TGF- $\beta 1 /$ Smad2/3 pathway. J Pharm Pharmacol 71: 1017-1028, 2019.

21. Liao WI, Wu SY, Wu GC, Pao HP, Tang SE, Huang KL and Chu SJ: Ac2-26, an Annexin A1 peptide, attenuates ischemia-reperfusion-induced acute lung injury. Int J Mol Sci 18: 1771, 2017.

22. Hung KY, Liao WI, Pao HP, Wu SY, Huang KL and Chu SJ: Targeting F-box protein Fbxo3 attenuates lung injury induced by ischemia-reperfusion in rats. Front Pharmacol 10: 583, 2019.

23. Kang H, Lee Y, Bae M, Park YK and Lee JY: Astaxanthin inhibits alcohol-induced inflammation and oxidative stress in macrophages in a sirtuin 1-dependent manner. J Nutr Biochem 85: $108477,2020$.

24. Yabuki H, Watanabe T, Oishi H, Katahira M, Kanehira M and Okada Y: Muse cells and ischemia-reperfusion lung injury. Adv Exp Med Biol 1103: 293-303, 2018.

25. Kreisel D and Goldstein DR: Innate immunity and organ transplantation: Focus on lung transplantation. Transpl Int 26: 2-10, 2013.

26. Rognlien AGW, Wollen EJ, Atneosen-Asegg M, Suganthan R, Bjoras M and Saugstad OD: Neonatal Ogg1/Mutyh knockout mice have altered inflammatory gene response compared to wildtype mice in the brain and lung after hypoxia-reoxygenation. J Perinat Med 47: 114-124, 2018.

27. Liu PL, Chong IW, Lee YC, Tsai JR, Wang HM, Hsieh CC, Kuo HF, Liu WL, Chen YH and Chen HL: Anti-inflammatory effects of resveratrol on Hypoxia/Reoxygenation-induced alveolar epithelial cell dysfunction. J Agric Food Chem 63: 9480-9487, 2015

28. Xie W, Zhou P, Sun Y, Meng X, Dai Z, Sun G and Sun X: Protective effects and target network analysis of ginsenoside Rg1 in cerebral ischemia and reperfusion injury: A comprehensive overview of experimental studies. Cells 7: 270, 2018

29. Sun $Z$ and Wang X: Protective effects of polydatin on multiple organ ischemia-reperfusion injury. Bioorg Chem 94: 103485, 2020.

30. Ye J, Lu S, Wang M, Ge W, Liu H, Qi Y, Fu J, Zhang Q, Zhang B, Sun G and Sun X: Hydroxysafflor yellow a protects against myocardial ischemia/reperfusion injury via suppressing NLRP3 inflammasome and activating autophagy. Front Pharmacol 11: $1170,2020$.

31. Luczkiewicz P, Kokotkiewicz A, Dampc A and Luczkiewicz M: Mangiferin: A promising therapeutic agent for rheumatoid arthritis treatment. Med Hypotheses 83: 570-574, 2014.

32. Liu K, Wang F, Wang S, Li WN and Ye Q: Mangiferin attenuates myocardial ischemia-reperfusion injury via MAPK/Nrf-2/HO-1/NF- $\kappa$ B in vitro and in vivo. Oxid Med Cell Longev 2019: 7285434, 2019.

33. Li N, Xiong R, He R, Liu B, Wang B and Geng Q: Mangiferin mitigates lipopolysaccharide-induced lung injury by inhibiting NLRP3 inflammasome activation. J Inflamm Res 14: 2289-2300, 2021.

34. Wang B, Wan J, Gong X, Kuang G, Cheng X and Min S: Mangiferin attenuates renal ischemia-reperfusion injury by inhibiting inflammation and inducing adenosine production. Int Immunopharmacol 25: 148-154, 2015.

35. Du M, Wen G, Jin J, Chen Y, Cao J and Xu A: Mangiferin prevents the growth of gastric carcinoma by blocking the PI3K-Akt signalling pathway. Anticancer Drugs 29: 167-175, 2018.

36. Xia G, Li X, Zhu X, Yin X, Ding H and Qiao Y: Mangiferin protects osteoblast against oxidative damage by modulation of ERK5/Nrf2 signaling. Biochem Biophys Res Commun 491: 807-813, 2017.

37. Jiang T, Han F, Gao G and Liu M: Mangiferin exert cardioprotective and anti-apoptotic effects in heart failure induced rats. Life Sci 249: 117476, 2020

38. Chen L, Li S, Zhu J, You A, Huang X, Yi X and Xue M: Mangiferin prevents myocardial infarction-induced apoptosis and heart failure in mice by activating the Sirt1/FoxO3a pathway. J Cell Mol Med 25: 2944-2955, 2021.

39. Chen M, Wang Z, Zhou W, Lu C, Ji T, Yang W, Jin Z, Tian Y, Lei $\mathrm{W}$, Wu S, et al: SIRT1/PGC-1 $\alpha$ signaling activation by mangiferin attenuates cerebral hypoxia/reoxygenation injury in neuroblastoma cells. Eur J Pharmacol 907: 174236, 2021.

40. Michan S and Sinclair D: Sirtuins in mammals: Insights into their biological function. Biochem J 404: 1-13, 2007. 
41. Liu S, Xu J, Fang C, Shi C, Zhang X, Yu B and Yin Y: Over-expression of heat shock protein 70 protects mice against lung ischemia/reperfusion injury through SIRT1/AMPK/eNOS pathway. Am J Transl Res 8: 4394-4404, 2016.

42. Li D, Wang X, Huang Q, Li S, Zhou Y and Li Z: Cardioprotection of CAPE-ONO ${ }_{2}$ against myocardial ischemia/reperfusion induced ROS generation via regulating the SIRT1/eNOS/NF- $\kappa$ B pathway in vivo and in vitro. Redox Biol 15: 62-73, 2018.

43. Ding S, Liu D, Wang L, Wang G and Zhu Y: Inhibiting microRNA-29a protects myocardial ischemia-reperfusion injury by targeting SIRT1 and suppressing oxidative stress and NLRP3-mediated pyroptosis pathway. J Pharmacol Exp Ther 372: 128-135, 2020.
44. Han Y, Sun W, Ren D, Zhang J, He Z, Fedorova J, Sun X, Han F and Li J: SIRT1 agonism modulates cardiac NLRP3 inflammasome through pyruvate dehydrogenase during ischemia and reperfusion. Redox Biol 34: 101538, 2020.

45. Lempiainen J, Finckenberg P, Levijoki J and Mervaala E: AMPK activator AICAR ameliorates ischaemia reperfusion injury in the rat kidney. Br J Pharmacol 166: 1905-1915, 2012.

(i) $(9)$ This work is licensed under a Creative Commons c. International (CC BY-NC-ND 4.0) License. 